【电子与信息科学 / Electronics and Information Science】

\title{
基于标签传播的协同分类欺诈检测方法
}

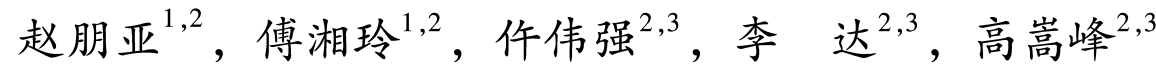

1) 北京邮电大学计算机学院 (国家示范性软件学院), 北京邮电大学可信分布式计算与服务教育部重点实验室, 北京 $100876 ； 2$ ) 北邮-华融智慧金融联合实验室，北京 $100876 ； 3$ ）华融融通（北京）科技有限公司，北京 100033

摘 要：网络借贷领域中的欺诈检测是根据收集到的用户历史交易数据等信息，来判断该用户是欺诈 用户还是正常用户. 现有方法认为用户是独立存在的，忽略了用户之间的关联信息. 考虑到目前欺诈逐渐 成为群体行为，在欺诈网络内呈现出欺诈节点与非欺诈节点关联稀疏，而欺诈节点间关联紧密的现象，提 出基于标签传播的协同分类欺诈检测方法. 通过收集真实网上借贷公司的用户通话数据，构建用户之间的 通话关联网络，利用标签传播算法扩散欺诈节点的标签信息，确定未知标签节点是否为欺诈用户。通过对 权重进行幂操作，改进了标签传播算法中概率转移矩阵的初始化方法，使其适应欺诈场景下正负样本分布 不平衡的现象. 在有标签样本比例极低且训练样本分布不均衡的真实借贷数据集中进行了 7 次测试, 采用 所提算法检测到欺诈用户的精确率最高达 $17 \%$ ，所得 $F_{1}$ 值与精确率都比经典的 WvRn 算法更优.

关键词：计算机软件；欺诈检测；协同分类；网络借贷；标签传播；机器学习

中图分类号：TP311; TP312 文献标志码：A doi：10.3724/SP. J. 1249.2020.05482

\section{Collective classification method based on label propagation for fraud detection}

\author{
ZHAO Pengya ${ }^{1,2}$, FU Xiangling ${ }^{1,2}$, WU Weiqiang ${ }^{2,3}$, \\ LI $\mathrm{Da}^{2,3}$, and GAO Songfeng ${ }^{2,3}$
}

1) School of Computer Science ( National Pilot Software Engineering School), Key Laboratory of Trustworthy

Distributed Computing and Service (BUPT), Ministry of Education, Beijing University of

Posts and Telecommunications, Beijing 100876, P. R. China

2) BUPT and Huarong Joint Lab of Smart Finance, Beijing 100876, P. R. China

3 ) Huarong Rongtong (Beijing) Technology Co. , Ltd. , Beijing 100033, P. R. China

\begin{abstract}
In the field of online lending, the key problem for fraud detection is how to judge whether the user is a fraudster or a normal user based on the collected historical transaction data of the user. At present, the representative research methods treat any user as an independent node and ignore the related information among users. Considering that the fraud is gradually becoming a group behavior, the relationships among fraud nodes and non-fraud nodes are sparse in social networks, and the relationships among fraud nodes are closely related, we propose a collective classification fraud detection method with label propagation. A call-records-based user association network is constructed based on the phone call records between users of online lending company, and we use the label propagation algorithm to spread the label information of fraud node to determine whether the unlabeled node is a
\end{abstract}

Received: 2019-04-28; Revised: 2019-06-04; Accepted: 2019-06-06

Foundation: National Natural Science Foundation of China (91546121); National Social Science Foundation of China (16ZDA055)

Corresponding author: Professor FU Xiangling. E-mail: fuxiangling@ bupt.edu.cn

Citation: ZHAO Pengya, FU Xiangling, WU Weiqiang, et al. Collective classification method based on label propagation for fraud detection $[\mathrm{J}]$. Journal of Shenzhen University Science and Engineering, 2020, 37 (5): 482-489. (in Chinese) 
fraudulent user. In addition, we improve the initialization method of transition probability matrixin label propagation algorithm by the operation of weights powering to avoid the performance degradation of label propagation algorithm caused by the unbalanced distribution of fraud data. Finally, the validation experiment is conducted in a real loan data set with a very low proportion of labeled samples and unbalanced training sample distribution. By using the proposed method in this article, the accuracy rate of fraud user detection reaches $17 \%$, and the $F_{1}$ value and accuracy rate are both better than those of the classic WvRn algorithm.

Key words: computer software; fraud detection; collective classification; online lending; label propagation; machine learning

欺计风险已成为当前消费金融公司面对的主要 风险，据 2018 年发布的《数字金融反欺诈白皮 书》，恶意欺诈产生的损失占消费金融公司整体坏 账的 $60 \%$. 从传统的采用黑名单 ${ }^{[1]}$ 和专家规则 ${ }^{[2]}$, 到如今的基于大数据的机器学习 (machine learning, $\mathrm{ML})$ ，这些欺诈检测 ${ }^{[3-5]}$ 方法都是根据申请时的注 册名称是否满足一定的模式，申请时使用的网际互 连协议 (internet protocol, IP) 号段是否为临时 IP 等, 来获得发现欺计者的非典型特征. 这些方案假设每 个用户之间独立存在，只考虑用户本身的固有特 征，未考虑用户在网络中的关联性. 目前网络借贷 场景下，一方面，随着大数据的发展，收集到的数 据维度增加，覆盖包括设备信息、IP 信息和通话信 息等, 使得研究人员能基于数据构建关联网络; 另 一方面, 欺诈行为在社交网络中体现出一种同质效

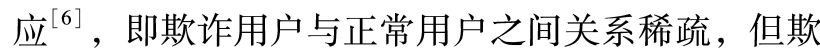
计用户之间关系紧密. 若某用户和多个欺计用户联 系密切，则该用户很大概率也是欺计者.

协同分类问题是指给定一个网络和部分节点的 标签信息, 如何根据网络信息推理出未知节点的标 签信息. 本研究提出基于标签传播的协同分类欺计 检测方法. 该方法基于关联网络，利用改进的标签 传播算法得到网络中未标记节点的欺计信息从而发 现欺计节点, 如图 1. 在由真实的网络借贷数据构 建的网络中进行实验, 证明这种以关联网络为基础 的网络标签传播方法在欺计检测中效果明显.

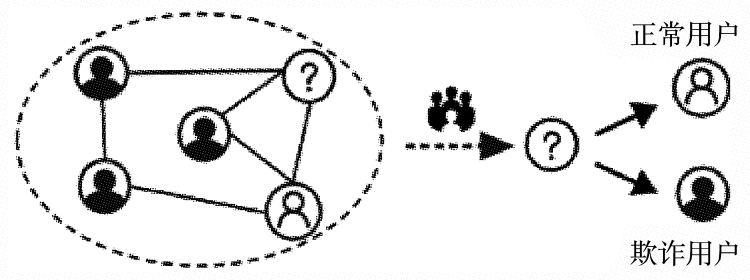

图 1 传播示例图

Fig. 1 An illustrative example of propagation

\section{1 研究背景}

\section{1 欺诈检测模型}

目前网络借贷领域下采用的欺计检测模型主要 分为 3 类: 逻辑回归 ${ }^{[7-9]}$ 、神经网络 ${ }^{[10-12]}$ 和基于树 算法的集成模型 ${ }^{[13-15]}$. 其中，逻辑回归因其对变量 的良好解释性，在检测初期得到了很好的应用，如 利用基于逻辑回归的评分卡模型获得用户的信用 值，再根据信用值的大小来调整贷款额度的多少. 但随着数据维度和特征数量的增加, 逻辑回归对于 非线性关系的拟合不够精准的缺陷逐渐显现. 神经 网络极大地提高了欺诈检测效果，但因网络本身是 个黑盒模型，而借贷领域更希望检测结果是可解释 的，因此，目前借贷领域中的主流模型更多是基于 树算法的集成模型, 如随机森林和 lightGBM (light gradient boosting machine) 等.

\section{2 协同分类}

上述机器学习模型大部分仍是监督学习模式, 在训练集上训练分类器, 在测试集上使用评价指标 评价模型性能. 此过程中训练集和测试集是分开 的, 且每个训练样本独立存在, 分类是在测试样本 上独立执行，未考虑样本之间潜在的网络关系. 网 络借贷中的欺诈行为常呈团体化趋势，即欺计者之 间具有聚集性和紧密关联现象，而欺计者和正常用 户之间则呈现分散性和稀疏关联.

首先给出关于协同分类问题的一些定义和符号 表达. 假设图结构 $G=(V, E)$. 其中, $V$ 为节点集合， $V_{\mathrm{L}}=\left\{v_{1}, v_{2}, \cdots, v_{n}\right\}$ 为已知标签的节点集合, $Y_{\mathrm{L}}=$ $\left\{y_{1}, y_{2}, \cdots, y_{n}\right\}$ 为已知标签节点的标签集合, $V_{\mathrm{U}}=$ $\left\{v_{n+1}, v_{n+2}, \cdots, v_{n+m}\right\}$ 为未知标签的节点集合, $Y_{\mathrm{U}}=$ $\left\{y_{n+1}, y_{n+2}, \cdots, y_{n+m}\right\}$ 为未知标签的预测标签值集 合; $E$ 为边的集合. $e_{i j}$ 为节点 $v_{i}$ 和节点 $v_{j}$ 之间的连接 边, $e_{i j} \in E, v_{i}, v_{j} \in V, w_{i j}$ 为边 $e_{i j}$ 的权重. $N(i)$ 为节点 $v_{i}$ 的邻居节点集合, $A(i)$ 为节点 $v_{i}$ 的属性集合. $L=$ 
$\left\{l_{1}, l_{2}, \cdots, l_{k}\right\}$ 为网络中所有的标签集合. 协同分 类问题认为网络中的一个未知标签节点 $\left(v_{i} \in V_{\mathrm{U}}\right)$, 其预测标签 $\left(y_{i} \in Y_{\mathrm{U}}\right)$ 受到 3 个因素影响: (1) 节点 自身的属性 $A(i)$; (2) 其邻居节点的属性 $A(j), v_{j} \in$ $N(i)$; (3) 其邻居节点的标签 $y_{j} \in Y_{\mathrm{L}}, v_{j} \in N(i)$.

协同分类就是利用上述因素对未知标签的节点 进行推理分类. 对于任意网络, 达到真正的推理分 类都是一个非确定性多项式 (non-deterministic polynomial，NP) 问题，因此，实际使用的协同分类大多 是迭代式的，本研究使用的标签传播算法亦是通过 迭代到收玫状态达到一种近似推理. 主流的协同分 类算法包括以下 3 类.

\subsection{1 关系分类算法}

关系分类 (relational classification, RC) 的主要方 法之一是带权表决近邻分类器 (weighted-vote relational neighbor classifier, WvRn $)^{[16]}$. 该方法认为一 个节点的标签仅由其邻居的标签决定，如式 (1), 先计算节点 $v_{i}$ 属于每个标签的概率，再取概率最大 值的标签作为其标签.

$$
P\left(y_{i}=l \mid N(i)\right)=\frac{1}{Z} \sum_{v_{j} \in N(i)} w_{i j} P\left(x_{j}=l\right)
$$

其中, $N(i)$ 为节点 $v_{i}$ 的邻居节点; $w_{i j}$ 为边 $e_{i j}$ 的权重; $Z=\sum_{v_{j} \in N(i)} w_{i j} ; l$ 为标签集合 $L$ 中的任一标签.

\subsection{2 迭代分类算法}

迭代分类算法 ${ }^{[17]}$ ( iterative classification algorithm, ICA) 分为初始化 (bootstrap) 和迭代分类 (iterative classification)两个过程，前者负责初始化节点 标签，后者负责迭代化更新节点标签. ICA 算法具 体步骤如图 2 .

bootstrap 过程

1) 对每个 $v_{i} \in V_{\mathrm{U}}, i=1,2, \cdots, m$, 执行步骤 2) 和 3);

2) 根据 $v_{i}$ 的属性及 $N(i)$ 中有标签的节点信息将 $v_{i}$ 用特 征向量 $\boldsymbol{a}_{i}$ 表示;

$3)$ 使用局部分类器 $M_{\mathrm{L}}$, 根据 $\boldsymbol{a}_{i}$ 的分类结果得到 $y_{i}$, 即 $y_{i} \leftarrow M_{\mathrm{L}}\left(\boldsymbol{a}_{i}\right)$;

iterative classification 过程

4) 打乱 $V_{\mathrm{U}}$ 顺序随机生成序列 $O$, 对于每个 $v_{i} \in O, i=$ $1,2, \cdots, m$, 执行步骤 5) 和 6$)$;

$5 ）$ 根据 $v_{i}$ 当前 $N(i)$ 的标签信息重构特征向量 $\boldsymbol{a}_{i}$;

6) 使用局部分类器 $M_{\mathrm{L}}$, 更新 $y_{i}$;

7）重复步骤 4）、5）和 6）, 直到所有的节点标签不再 变化或达到最大迭代次数.

图 2 ICA 算法步骤

Fig. 2 Process of ICA algorithm

\subsection{3 吉布斯采样}

吉布斯采样 ${ }^{[18]}$ (Gibbs sampling) 分为 bootstrap、 加热 (burn-in) 和采样 ( sample) 过程, 具体步骤如 图 3.

\section{bootstrap 过程}

1) 对每个 $v_{i} \in V_{\mathrm{U}}, i=1,2, \cdots, m$, 执行步骤 2) 和 3$)$;

2）根据 $v_{i}$ 的属性及 $N(i)$ 中有标签的节点信息，将 $v_{i}$ 用 $a_{i}$ 表示;

$3)$ 根据 $\boldsymbol{a}_{i}$ 的分类结果得到 $y_{i}$, 即 $y_{i} \leftarrow M_{\mathrm{L}}\left(\boldsymbol{a}_{i}\right)$; burn in 过程 (不进行统计操作)

4) 打乱 $V_{\mathrm{U}}$ 顺序随机生成序列 $O$, 对于每个 $v_{i} \in O, i=1$, $2, \cdots, m$, 都执行步骤 5) 和 6);

$5 ）$ 根据节点 $v_{i}$ 当前 $N(i)$ 的标签信息重构 $\boldsymbol{a}_{i}$;

6) 用局部分类器 $M_{\mathrm{L}}$ 更新 $y_{i}$;

sample 过程（进行统计操作）

7）针对每个节点 $v_{i} \in V_{\mathrm{U}}$ ，初始化 $c[i, l]=0$;

8) 打乱 $V_{\mathrm{U}}$ 顺序随机生成序列 $O$, 对于每个 $v_{i} \in O, i=1$, $2, \cdots, m$, 都执行步骤 9)-12);

9) 根据 $v_{i}$ 当前的 $N(i)$ 的标签信息重新构建特征向量 $\boldsymbol{a}_{i} ;$

10) 使用局部分类器 $M_{\mathrm{L}}$ 更新 $y_{i}$, 并更新 $c\left[i, l_{i}\right] \leftarrow c[i$, $\left.l_{i}\right]+1 ;$

11）返回执行步骤 8）、9）和 10），直至达到迭代次数; 12）针对每个节点 $v_{i} \in V_{\mathrm{U}}, y_{i} \leftarrow \operatorname{argmax}_{c \in L} c[i, l]$.

图 3 吉布斯采样步骤

Fig. 3 Process of Gibbs sampling

\section{3 标签传播算法}

标签传播算法 ${ }^{[19]}$ ( label propagation algorithm, LPA）是一种基于图的半监督学习算法，其主要思 想是基于相关网络的前提预设，利用少量的有标签 数据标记待预测数据. 标签传播的假设前提是：邻 近的样本点更可能拥有相同标签. 这里，衡量邻近 与否的可以是图中两个点的欧式距离，也可以是网 络中代表点之间关联度的边的权重. 比如，通话关 系网络中，边的权重代表了两用户之间的通话密切 程度. 本研究认为, 关联度较高的两个点更可能拥 有相同标签. 标签传播算法具有很强的通用性，对 于符合其假设前提的问题场景，能够利用少量已标 注节点预测未标记节点的标签信息，将标签传播至 
未标注节点. 考虑到欺计在网络内呈现的特性符合 标签传播的基本假设前提，使用标签传播来将已确 认的欺计节点信息扩散开来，获取其余未标注节点 的标签信息, 辅助进行欺计检测.

有学者将标签传播引入到欺计检测中. PENG 等 ${ }^{[20]}$ 抽取通话记录转化成网络, 使用标签传播进 行社区发现, 并进行欺计社区的发现, 实现了快速 分辨欺计电话, 研究使用标签传播旨在进行社区检 测. CUI 等 ${ }^{[21]}$ 将标签传播应用到医保欺计检测领 域，通过凸凹变换，将凸标签传播算法转变为稀有 标签的非凸标签传播, 从医保数据集中识别存在欺 诈可能性的异常记录.

\section{2 关联网络的构建与分析}

\section{1 构建数据集}

为验证算法在真实环境下的有效性, 使用真实 的网络借贷数据构建网络. 数据集主要包含 3 部分 数据: (1) 职业数据, 包括用户所属的行业信息, 如金融和医疗等; (2) 应用 (application, app)列表数 据, 即用户移动设备上安装的 app 列表, 经过脱敏 处理, 每个 app 上使用唯一的数字标识; (3) 通话数 据记录两用户之间的通话情况, 包含当月通话次数 和通话权重.

实验采集到的原始数据集包含 246 万个用户相 关数据. 其中, 18405 个用户为有标签用户, 即确 定是欺计用户或正常用户, 具体分布为 2782 个欺 许用户，15623 个正常用户。

\section{2 数据抽取}

为了能够更直观地体现算法的有效性, 抽取原 数据集中的通话数据(数据格式如表 1 ), 以用户身 份标识号 (identity document, ID) 为节点, 用户之间 的通话关系为边构建关联网络. 其中, 时间窗口为 2017 年 12 月; $T$ 为当月通话次数; $t$ 为当月通话时 间; $w$ 为权重，且 $w=T t$.

表 1 通话关系格式

Table 1 The sample of call data

\begin{tabular}{ccccc}
\hline $\mathrm{ID}_{1}$ & $\mathrm{ID}_{2}$ & $T /$ 次 & $t / \min$ & $w$ \\
\hline 10000519 & 8666414 & 37 & 74 & 2738 \\
10000019 & 23264041 & 1 & 11 & 11 \\
$\vdots$ & $\vdots$ & $\vdots$ & $\vdots$ & $\vdots$ \\
\hline
\end{tabular}

需要注意的是, 通话数据集中的通话关系是一 种有向关系, 即用户 $A$ 打电话给用户 $B$, 和用户 $B$ 打电话给用户 $A$ 是不同的. 具体到要构建的图中, 则表现为两点之间可能存在一条单向边或两条有向 边的情况. 本研究认为两个用户之间的通话关系是 互相的，即用户 $A$ 对用户 $B$ 的多次通话能证明用户 $B$ 和用户 $A$ 之间关系亲密. 因此, 采取如下操作构 建网络的边：(1) 若两点之间只有单向边, 则直接 转化为无向图, 且保持原权重; (2) 若两点之间存 在双向边, 则将两条边的权重相加, 将两条有向边 转化为一条无向边, 并将之前得到的权重赋给该 边. 采用此方法, 最终构成的无向有权重通话网络 包含 2462611 个节点和 2709492 条边.

为评价构建的通话关联网络是否适于本研究使 用的标签传播进行协同分类, 以下从两方面对通话 关联网络分析.

\section{3 连通性}

若网络的连通性太低, 虽然网络节点很多, 但 由于它是由很多个小的连通子图构成, 依然会对最 后的标签传播效果造成影响. 本研究采用式 (2) 的 $C(G)$ 指标来衡量本研究构建的通话关联网络 $G$ 的 连通性.

$$
\begin{aligned}
& C(G)=\frac{\text { 最大连通分量节点数目 }}{\text { 全部节点数目 }} \\
& \text { 由式(2)可得, } C(G)=\frac{2327676}{2462611}=0.94 . \text { 可 }
\end{aligned}
$$

见, 本研究构建的网络的整体连通性高, 有利于标 签传播的应用.

\section{4 标签传播前提验证}

LPA 的假设前提等价于网络的同质性, 如式 (3), 即若两个节点的标签相同, 则两节点之间有 连接边的概率更大.

$$
P\left(A \rightarrow B \mid A_{\mathrm{L}}=B_{\mathrm{L}}\right)>P(A \rightarrow B)
$$

其中, $P(A \rightarrow B)$ 为节点 $A$ 和节点 $B$ 之间有边连接的 概率; $A_{\mathrm{L}}$ 和 $B_{\mathrm{L}}$ 分别代表节点 $A$ 和节点 $B$ 的标签.

为验证通话网络的同质性, 采用常用的标签一 致性 ${ }^{[21]}$ 、同类亲密度 ${ }^{[22]}$ 和异类亲密度 ${ }^{[22]}$ 指标来衡 量网络同质性. 其中, 标签一致性描述网络中连接 的两个节点标签相同的边占全部边的比例, 该值小 于 1 , 且值越大表示网络的同质性越强; 同类亲密 度描述欺计节点之间的关联紧密程度, 该值大于 1 , 且值越大表示与随机网络相比, 该网络中欺计节点 之间的关联越紧密; 异类亲密度描述欺计与非欺诈 
节点之间的关联密度，该值小于 1 ，且值越小表示 与随机网络相比, 欺计与非欺计节点的关联越稀 疏. 基于上述指标的计算方式，得到关联网络的标 签一致性为 0.924 , 同类亲密度为 3.679 , 异类亲 密度为 0.295 . 可见, 本研究构建的通话网络满足 同质特性，符合标签传播的假设前提.

\section{3 算法及改进}

\section{1 LPA 算法的实现}

LPA 主要分为构建概率转移矩阵 $\boldsymbol{P}$ 和标注矩阵 $F$ 两部分，利用矩阵计算重复传播，最后达到收玫. LPA 具体流程如图 4.

输入: 构建关联网络, 其中 $l$ 个已确定标签节点, $u$ 个未 知标签节点, $C$ 个标签类别

输出 : $u$ 个未知标签节点的标签概率分布

1) 设置停止标签传播的迭代次数 $t_{\text {max }}$, 变化阈值 $\delta$.

2) 根据边的权重, 得到维度为 $(l+u) \times(l+u)$ 的邻接 权重矩阵 $W$, 其元素 $W_{i j}$ 为节点 $i$ 和节点 $j$ 的权重.

3) 利用式 (4) 将 $\boldsymbol{W}$ 按行进行归一化处理，转化为概率 转移矩阵 $\boldsymbol{P}=\left(P_{i j}\right)$

$$
P_{i j}=P(i \rightarrow j)=\frac{w_{i j}}{\sum_{k=1}^{l+u} w_{i k}}
$$

4) 初始化一个维度为 $(l+u) \times C$ 的标签概率分布矩阵 $F$. 首先, 确定 $l$ 个有标签样本的 $l \times C$ 矩阵 $Y_{\mathrm{L}}$, 其第 $i$ 行表示第 $i$ 个样本的标签概率向量, 即若第 $i$ 个样本 的类别是 $j$, 则该行第 $j$ 个元素为 1 , 其他元素为 0 . 同 样, 给 $u$ 个无标签样本一个维度为 $u \times C$ 的标注矩阵 $Y_{\mathrm{U}}$. 将 $Y_{\mathrm{L}}$ 和 $Y_{\mathrm{U}}$ 合并, 得到 $F=\left[\begin{array}{l}Y_{\mathrm{L}} \\ Y_{\mathrm{U}}\end{array}\right]$.

5）每个节点按照转移概率将它周围节点传播的标注值 按照权重相加，并更新自己的标签概率分布

$F_{i j}=\sum_{k=1}^{l+u} P_{i k} F_{j k}, 1 \leqslant i \leqslant l+u ; 1 \leqslant j \leqslant C$

6）限定已确定标签样本的概率分布，把标签概率分布 恢复为初始值

$F_{i j}=Y_{i j}, 1 \leqslant i \leqslant l ; 1 \leqslant j \leqslant C$

7 ) 重复步骤 5$)$ 和步骤 6$)$, 计算 $\boldsymbol{F}$ 相对于上次的改变量

$\Delta=\sum_{i=1}^{l+u} \sum_{j=1}^{l+u}\left(F_{i j}^{\mathrm{new}}-F_{i j}^{\mathrm{old}}\right)$

8）迭代次数达到 $t_{\text {max }}$, 或者 $\Delta<\delta$, 算法结束.

图 4 标签传播算法框架图

Fig. 4 Process of LPA

\section{2 不平衡标签传播算法}

由于欺诈数据集有类别不平衡现象，即欺诈样 本的数量比正常样本的少，直接使用 LPA，其性能 显著下降. 为使算法更适合当前数据集，本研究通 过改进 LPA，提出不平衡标签传播算法 ( unbalanced label propagation algorithm, ULPA).

本研究采用类似图像处理中的小波变换 ${ }^{[23]}$ 思 路, 增强权重值较大的, 削弱权重值较小的. 对初 始邻接矩阵的每个权重元素 $W$ 作如式 (8) 的非线性 变化.

$$
W_{\text {new }}=\left(W_{\text {old }}\right)^{n}
$$

其中, $W_{\text {old }}$ 为原始的标签传播的初始邻接权重; $W_{\text {new }}$ 为改进后的权重; $n$ 为幂的指数, 本实验分别设为 $1 、 2 、 3 、 4 、 5$. 每次实验迭代 500 次，得到对应 的 $F_{1}$ 值 ( $F$-score) 、曲线下面积 ( area under curve AUC) 和精确率 $P_{\text {fraud }}$ 结果如表 2 .

表 2 不同 $n$ 下的实验结果 ${ }^{1)}$

Table 2 Result of different $n$

\begin{tabular}{cccc}
\hline$n$ & $F_{1}$ & AUC & $P_{\text {fraud }} / \%$ \\
\hline 1 & 0.12 & 0.85 & 8 \\
2 & 0.19 & 0.87 & 15 \\
3 & 0.20 & 0.86 & 17 \\
4 & 0.18 & 0.82 & 13 \\
5 & 0.15 & 0.80 & 10 \\
\hline
\end{tabular}

1)带阴影数值为最优值

由表 1 可见，求幂后，3 种评价指标都比直接 将权重放人转移矩阵 $(n=1)$ 时更好, 且当 $n=3$ 时综合效果最好, $F_{1}=0.20, P_{\text {fraud }}=17 \%$, 皆为最 佳值, 而 AUC 也能达到较优值. 因此, 后续实验 取 $n=3$.

\section{4 实验与验证}

算法输出的标签概率分布, 实际上是用户的软 标签 $L_{\text {soft }}$. 采用式 (9) 将软标签转化为硬标签 $L_{\text {hard }}\left(L_{\text {hard }}=1\right.$ 表示该用户为欺计用户; $L_{\text {hard }}=0$ 表示 该用户为正常用户)，即类似给定 $L_{\text {soft }}=[a, b]$, $0 \leqslant a, b \leqslant 1, a+b=1$. 其中, $a$ 和 $b$ 分别代表欺计 和正常的概率.

$$
L_{\text {hard }}= \begin{cases}1, & a<b \\ 0, & a \geqslant b\end{cases}
$$


实验步骤为:

1）从关联网络中已确认标签中的节点中, 抽 取 2000 个作为测试节点;

2) 记录测试节点真实的标签后，抹掉标签， 则该节点变为未知标签的节点;

3) 使用改进型标签传播算法 ULPA;

4) 对测试节点的标签分布概率做硬标签转化 操作, 确认标签.

欺诈检测实质上是一个分类问题，旨在查找出 更多的欺计者且降低误杀率, 对应到机器学习中则 可用精确率 $P_{\text {fraud }}$ 表示: 假设抽取的测试节点通过标 签传播后, 预测为欺计的节点数为 $a$, 预测为欺计 且真实标签为欺计的节点数为 $b$, 则

$$
P_{\text {fraud }}=\frac{b}{a} \times 100 \%
$$

采用 $F_{1}$ 值和 AUC 综合评价最终的欺计检测结 果. 针对分类任务最后输出的连续变量, 设定多个 阈值, 从而计算出一系列阈值下的真正率和假正 率. AUC 值越大, 表示模型的预测结果越好. 7 次 试验下测试节点的标签分布状况以及最终预测结果 如表 3, 各评价指标结果如图 5 和图 6.

\section{表 3 多轮试验下的用户标签分布状况以及最终预测结果 \\ Table 3 User distribution and $\boldsymbol{P}_{\text {fraud }}$ under multiple rounds of testing}

\begin{tabular}{cccccc}
\hline $\begin{array}{c}\text { 测试 } \\
\text { 序号 }\end{array}$ & $\begin{array}{c}\text { 正常 } \\
\text { 用户数 }\end{array}$ & $\begin{array}{c}\text { 欺计 } \\
\text { 用户数 }\end{array}$ & $a$ & $b$ & $P_{\text {fraud }} / \%$ \\
\hline 1 & 1695 & 305 & 283 & 47 & 16.6 \\
2 & 1716 & 284 & 311 & 53 & 17.0 \\
3 & 1692 & 308 & 282 & 47 & 16.6 \\
4 & 1683 & 317 & 301 & 49 & 16.2 \\
5 & 1685 & 315 & 328 & 55 & 16.8 \\
6 & 1705 & 295 & 327 & 56 & 17.1 \\
7 & 1718 & 282 & 294 & 45 & 15.3 \\
\hline
\end{tabular}

从图 5 可见, 针对欺诈样本的精确率 $P_{\text {fraud }}$ 和 $F_{1}$ 值随迭代次数的增加而升高, 说明标签传播算法 有效，即随着迭代过程将标签节点的标签信息传播 到未标记的节点上, 改变了未标记节点的标签概率 分布, 进一步识别了欺计样本. 另外, 当迭代次数 超过 250 次后, 曲线变化已很小了, 甚至当迭代次

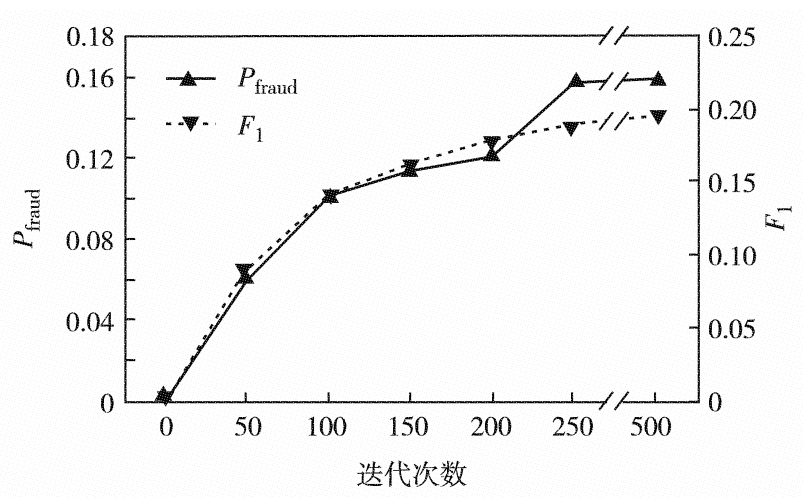

图 5 采用 ULPA 进行欺诈检测时不同迭代 次数下的 $P_{\text {fraud }}$ 和 $F_{1}$ 值

Fig. $5 P_{\text {fraud }}$ and $F_{1}$ from ULPA under different iterations

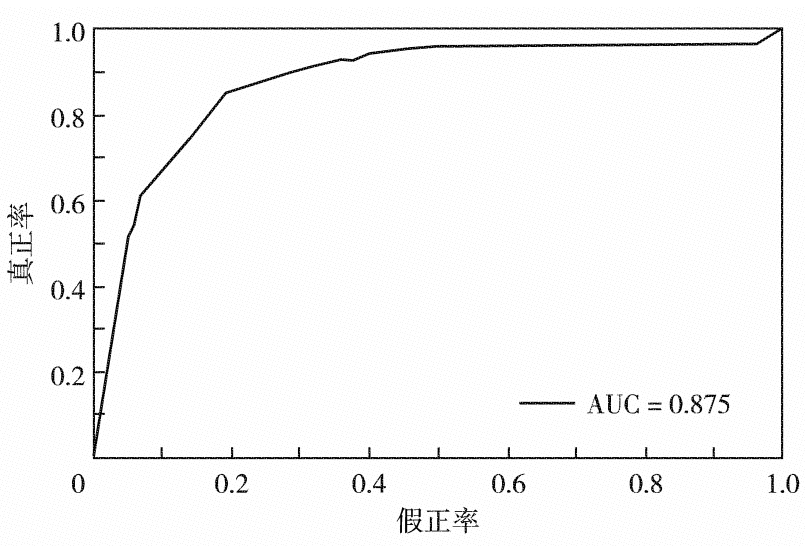

图 6 采用 ULPA 进行欺诈检测的

ROC 曲线和 AUC 值

Fig. 6 ROC curve and AUC from ULPA

数超过 500 次后, 曲线几乎不再发生变化, 原因是 数据集中有标签节点的比例太少, 标签传播很快达 到稳态.

图 6 统计了模型的接受者操作特性 (receiver operating characteristic, ROC) 曲线和 AUC 值. 由图 6 可见, 本研究模型的 AUC 指标较高, 说明模型预 测结果的区分性较高, 鲁棒性更好.

分别采用协同分类中经典的 $\mathrm{WvRn}$ 算法与本研 究算法进行欺计检测, 对比两种算法多次实验所得 $F_{1}$ 值和 $P_{\text {fraud }}$, 结果如图 7 和图 8.

由图 7 和图 8 可见, 使用本研究标签传播算法 得到的 $F_{1}$ 值和 $P_{\text {fraud }}$ 值都优于 $\mathrm{WvRn}$ 算法. 但是, 两种方法都存在多次实验下结果波动的现象，原因 是多次实验中, 每次实验随机选择的测试节点不 同. 此外, $\mathrm{WvRn}_{\mathrm{v}}$ 算法只考虑节点周围一度关联节 点的标签来确定自身的标签, 这种造成在有标签节 


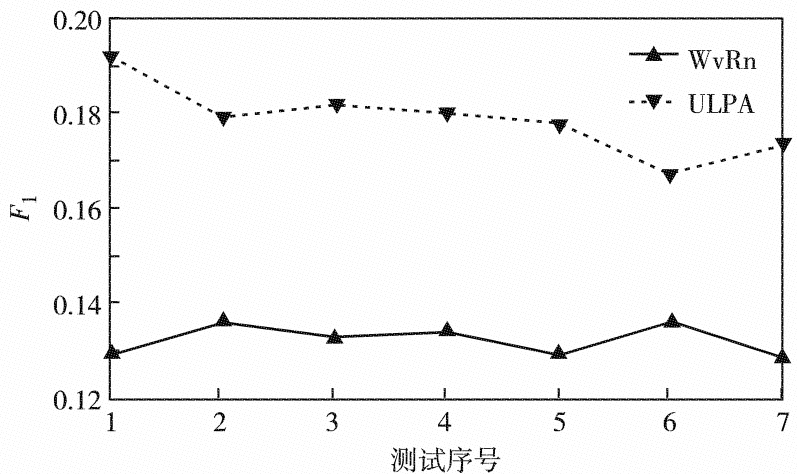

图 7 采用 WvRn 算法和 ULPA 进行 欺诈检测的 $F_{1}$ 值对比

Fig. $7 \quad F_{1}$ from comparison of $W v R n$ and

ULPA under multiple rounds of testing

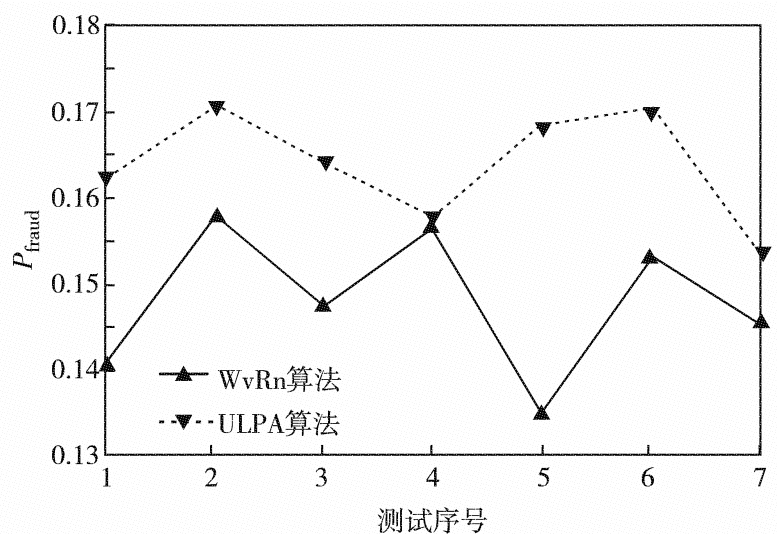

图 8 采用 WvRn 算法和 ULPA 进行 欺诈检测的 $\boldsymbol{P}_{\text {fraud }}$ 对比

Fig. $8 P_{\text {fraud }}$ from comparison of WvRn and

ULPA under multiple rounds of testing

点很稀疏的状况下，无法得出充分合理的标签推 断, 而本研究算法通过构建概率转移矩阵和标签分 布矩阵，采用迭代式的方法捕捉到多度关联节点的 标签信息，标签推理结果更符合真实场景.

\section{结 语}

本研究提出一种基于标签传播算法来进行协同 分类从而发现欺计者的方法，通过构建的通话网络 以及网络内已知的欺计节点, 将欺计信息通过标签 传播算法扩散开来，获得未知节点的标签概率分 布. 同时，为弥补因欺诈数据集中正负样本不均衡 导致的传播算法下降缺陷, 改进了概率转移矩阵的 权重设置初始化方法，改进之后相对于原始的标签
传播算法的 $F_{1}$ 指标从 0.12 提升到 0.20 ，提升了约 $67 \%$. 将该算法与经典的 $\mathrm{WvRn}$ 算法进行实验对 比，获得了更好的性能表现. 但是，本研究的标签 传播算法仅仅利用了网络的拓扑结构，虽然具有其 他任务领域的迁移性，但却无法有效利用其他用户 数据，因此后续可加人其他用户数据以辅助欺计信 息的传播检测.

基金项目：国家自然科学基金资助项目（91546121）；国家社会科 学基金资助项目 (16ZDA055)

作者简介：赵朋亚 (1995-) , 北京邮电大学硕士研究生。研究方 向：网络表征学习、欺计检测 E-mail: zpy1101936864@ bupt.edu.cn

引文：赵朋亚，傅湘玲，仵伟强，等．基于标签传播的协同分 类欺诈检测方法 $[\mathrm{J}]$. 深圳大学学报理工版, 2020,37 (5) : 482-489.

\section{参考文献 / References:}

[ 1 ] CALDERA J, HAIN J M, SHERLOCK K. Enhanced automated anti-fraud and anti-money-laundering payment system: U. S. Patent Application 14/846, 169 [ P ]. 2016-03-10.

[ 2 ] SARNO R, DEWANDONO R D, AHMAD T, et al. Hybrid association rule learning and process mining for fraud detection $[\mathrm{J}]$. IAENG International Journal of Computer Science, 2015, 42(2) : 59-72.

[ 3 ] WANG Hao, WANG Zonghu, ZHANG Bin, et al. Information collection for fraud detection in $\mathrm{P} 2 \mathrm{P}$ financial market $[\mathrm{C}] / /$ The 2nd International Conference on Material Engineering and Advanced Manufacturing Technology. [S. l. ] : EDP Sciences, 2018, 189: 06006.

[ 4 ] FERNANDEZ A. Artificial intelligence in financial services $[\mathrm{J}]$. Banco de Espana Article, 2019, 7: 19.

[ 5 ] AHMED M, MAHMOOD A N, ISLAM M R. A survey of anomaly detection techniques in financial domain $[\mathrm{J}]$. Future Generation Computer Systems, 2016, 55: 278 288.

[ 6 ] BAESENS B, Van VLASSELAER V, VERBEKE W. Fraud analytics using descriptive, predictive, and social network techniques: a guide to data science for fraud detection $[\mathrm{M}]$. Hoboken, USA: John Wiley \& Sons, Inc, 2015.

[ 7 ] ITOO F, Meenakshi, SINGH S. Comparison and analysis of logistic regression, Naïve Bayes and $k \mathrm{NN}$ machine learning algorithms for credit card fraud detection $[\mathrm{J}]$. International Journal of Information Technology. (202002-15 ). https ://doi. org/10. 1007/s41870-020-00430-y.

[ 8 ] SINGH N, LAI K H, VEJVAR M, et al. Data-driven 
auditing: a predictive modeling approach to fraud detection and classification $[\mathrm{J}]$. Journal of Corporate Accounting \& Finance, 2019, 30(3) : 64-82.

[ 9 ] CARNEIRO N, FIGUEIRA G, COSTA M. A data mining based system for credit-card fraud detection in e-tail $[\mathrm{J}]$. Decision Support Systems, 2017, 95: 91-101.

[10 ] FU Kang, CHENG Dawei, TU Yi, et al. Credit card fraud detection using convolutional neural networks $[\mathrm{C}] / /$ Proceedings of the 23th International Conference on Neural Information Processing. Kyoto, Japan: Springer, 2016: 483-490.

[11] ZAKARYAZAD A, DUMAN E. A profit-driven artificial neural network (ANN) with applications to fraud detection and direct marketing $[\mathrm{J}]$. Neurocomputing, 2016, 175 : 121-131.

[12] ZHANG Zhaohui, ZHOU Xinxin, ZHANG Xiaobo, et al. A model based on convolutional neural network for online transaction fraud detection $[\mathrm{J}]$. Security and Communication Networks, 2018(2): 1-9.

[13] SAVE P, TIWAREKAR P, JAIN K N, et al. A novel idea for credit card fraud detection using decision tree $[\mathrm{J}]$. International Journal of Computer Applications, 2017, 161 (13) : 6-9.

[14] XUAN Shiyang, LIU Guanjun, LI Zhenchuan, et al. Random forest for credit card fraud detection $[\mathrm{C}] / /$ The 15th International Conference on Networking, Sensing and Control (ICNSC). Zhuhai, China: IEEE, 2018: 1-6.

[15] KHARE N, VISWANATHAN P. Decision tree-based fraud detection mechanism by analyzing uncertain data in banking system [M]// Emerging Research in Data Engineering Systems and Computer Communications. Singapore: Springer, 2020: 79-90.

[16] MACSKASSY S A, PROVOST F. Classification in networked data: a toolkit and a univariate case study $[\mathrm{J}]$. Journal of Machine Learning Research, 2007, 8(1) : 935983.
[17] JENSEN D, NEVILLE J, GALLAGHER B. Why collective inference improves relational classification $[\mathrm{C}] / /$ Proceedings of the 10th ACM SIGKDD International Conference on Knowledge Discovery and Data Mining. Seattle, USA: Association for Computing Machinery, 2004: 593-598.

[18 ] MCDOWELL L K, GUPTA K M, AHA D W. Cautious inference in collective classification $[\mathrm{C}] / /$ Proceedings of the 22nd National Conference on Artificial Intelligence. Vancouver, Canada: AAAI, 2007: 596-601.

[19] GREGORY S. Finding overlapping communities in networks by label propagation $[\mathrm{J}]$. New Journal of Physics, 2010, 12(10): 103018.

[20] PENG Lu, LIN Rongheng. Fraud phone calls analysis based on label propagation community detection algorithm [C]// IEEE World Congress on Services. San Francisco, USA : IEEE, 2018: 23-24.

[21] CUI Haoyi, LI Qingzhong, LI Hui, et al. Healthcare fraud detection based on trustworthiness of doctors $[\mathrm{C}] / /$ IEEE International Conference on Trust, Security and Privacy in Computing and Communications. Tianjin, China: IEEE, 2016: 74-81.

[22] KOHLI P, LADICKY L, TORR P H S. Robust higher order potentials for enforcing label consistency $[\mathrm{J}]$. International Journal of Computer Vision, 2009, 82 (3) : 302324.

[23 ] PARK J, BARABÁSI A L. Distribution of node characteristics in complex networks $[\mathrm{J}]$. Proceedings of the National Academy of Sciences, 2007, 104(46) : 17916-17920.

[24] WONG C Y, LIU S, LIU S C, et al. Image contrast enhancement using histogram equalization with maximum intensity coverage $[\mathrm{J}]$. Journal of Modern Optics, 2016, 63(16) : 1618-1629.

【中文责编：英 子; 英文责编：木 柯】 